\title{
Te doping of GaAs using metalorganic vapor phase epitaxy: Volatile versus nonvolatile behavior
}

\author{
Beatriz Galiana, ${ }^{a)}$ Ignacio Rey-Stolle, Carlos Algora, and Iván García \\ Instituto de Energía Solar, Universidad Politécnica de Madrid ETSI de Telecomunicación, Avda. \\ Complutense s/n; 28040 Madrid, Spain
}

(Received 31 July 2008; accepted 14 October 2008; published online 8 December 2008)

\begin{abstract}
The incorporation of Te into the crystal lattice, when it is used as an $n$-type dopant for GaAs grown by metalorganic vapor phase epitaxy, is studied. For this purpose, several growth temperatures, total pressures, growth rates, and substrate misorientations have been analyzed, from which it is concluded that depending on the substrate misorientation and total pressure used, the Te behaves like a volatile dopant or a nonvolatile dopant as result of the enhancement or minimization of its adsorption onto the growth surface. () 2008 American Institute of Physics.
\end{abstract}

[DOI: $10.1063 / 1.3033398$ ]

\section{INTRODUCTION}

Tellurium has several remarkable properties that makes it an attractive $n$-type dopant for GaAs. ${ }^{1-5}$ It allows high doping levels to be achieved as result of a low activation energy compared to other typical $n$-type dopants such as silicon. ${ }^{5,6}$ It shows a weaker memory effect as compared to other group-VI dopants such as sulfur or selenium as well as having a low diffusion coefficient in $\mathrm{GaAs}^{5}$ that favors the growth of abrupt interfaces, which is a significant factor in the development of tunnel junctions. ${ }^{7}$ In the analysis of other $n$-type dopants for GaAs, we also found that silicon hardly diffuses in GaAs, but on the other hand, carrier concentrations of more than $5 \times 10^{18} \mathrm{~cm}^{-3}$ are very difficult to achieve since $\mathrm{Si}$ tends to compensate itself as result of its anfoterous nature. Other alternative dopants such as selenium ${ }^{5}$ can achieve very high concentration levels (more than 1 $\times 10^{20} \mathrm{~cm}^{-3}$ ) but shows a strong memory effect (typical of most group-VI dopants) and diffuses significantly in GaAs, ${ }^{8}$ making it less controllable than Te. Other options for $n$-type dopants in GaAs, although with fewer applications, include tin (similar to silicon but with fewer and more expensive precursors) ${ }^{5}$ and sulfur (which shows a strong memory effect)..$^{5}$

Consequently, the study and comprehension of Te incorporation into GaAs grown by metalorganic vapor phase epitaxy (MOVPE) is an interesting issue. Unfortunately, the growth of GaAs doped with Te by MOVPE has not been widely used, probably because of its surfactant nature, which adds an extra degree of complexity (and freedom) to the growth. This implies that small amounts of Te atoms modify the processes on the surface, ${ }^{3}$ introducing kinetic effects that are unusual in the typical regime for the growth of III-V compounds by MOVPE. For example, the influence of Te in the growth of GaInP has been previously studied ${ }^{9}$ concluding that depending on the amount of Te, the incorporation of In and $\mathrm{Ga}$ into the crystal lattice is altered, modifying the composition of GaInP as well as reducing its $\mathrm{CuPt}$ ordering. ${ }^{10} \mathrm{In}$

\footnotetext{
${ }^{a)}$ Electronic mail: beatriz@ies-def.upm.es. Tel.: + 3491 5441060. FAX: +34915446341.
}

the case of GaAs doped with Te, its surfactant effect has attracted some attention in the literature where several theoretical studies have been published, ${ }^{11}$ as well as other works relating $\mathrm{Te}$ surfactant action with the resultant epilayer morphology. ${ }^{12}$ However, its influence on dopant incorporation has not yet been studied.

Consequently, the purpose of this paper is to analyze the Te incorporation in GaAs when grown by MOVPE in order to predict and understand the trends in the carrier concentration when varying several growth parameters. With this aim, GaAs layers doped with Te have been grown by MOVPE for different growth temperatures $\left(T_{G}\right)$, growth rates $\left(G_{R}\right)$, total pressures $\left(P_{T}\right)$, and substrate misorientations.

\section{EXPERIMENTAL}

The MOVPE growths have been carried out in an AIX200 horizontal reactor at total pressures $\left(P_{T}\right)$ of 50 and 100 mbar. All samples were grown on semi-insulating GaAs wafers with two different orientations, namely, (100) substrates with a miscut of $2^{\circ}$ toward the nearest (111)A plane and (100) substrates with a miscut of $2^{\circ}$ toward the nearest $(1 \overline{1} 0)$. In order to facilitate the reading of the paper, hereinafter the substrates will be referred to only by their misorientation, i.e., $2^{\circ} \rightarrow(111) A$ or $2^{\circ} \rightarrow(1 \overline{1} 0)$, thus avoiding recurrent references to the main (100) orientation. The range of growth temperatures explored is from 600 to $675{ }^{\circ} \mathrm{C}$. The total flow $\left(F_{T}\right)$ has been set to 6000 SCCM (SCCM denotes standard cubic centimeter per minute at STP) for the growths carried out at $50 \mathrm{mbar}$ and $14000 \mathrm{SCCM}$ when the $P_{T}$ was 100 mbar. The carrier gas was Pd-purified $\mathrm{H}_{2}$ and the precursors used were trimethyl gallium (TMGa), $\mathrm{AsH}_{3}$, and diethyl telluride (DETe) diluted in $\mathrm{H}_{2}(200 \mathrm{ppm})$. The magnitude used in relation to the flow of the precursors, the molar fraction $([X])$, is defined as the ratio between the flow of the precursor $(\mathrm{mol} / \mathrm{min})$ and the total flow. In all the experiments, the analyzed magnitude has been the carrier concentration $\left(N_{D}\right)$. It has been measured by means of electrochemical capacitance-voltage profiling. 


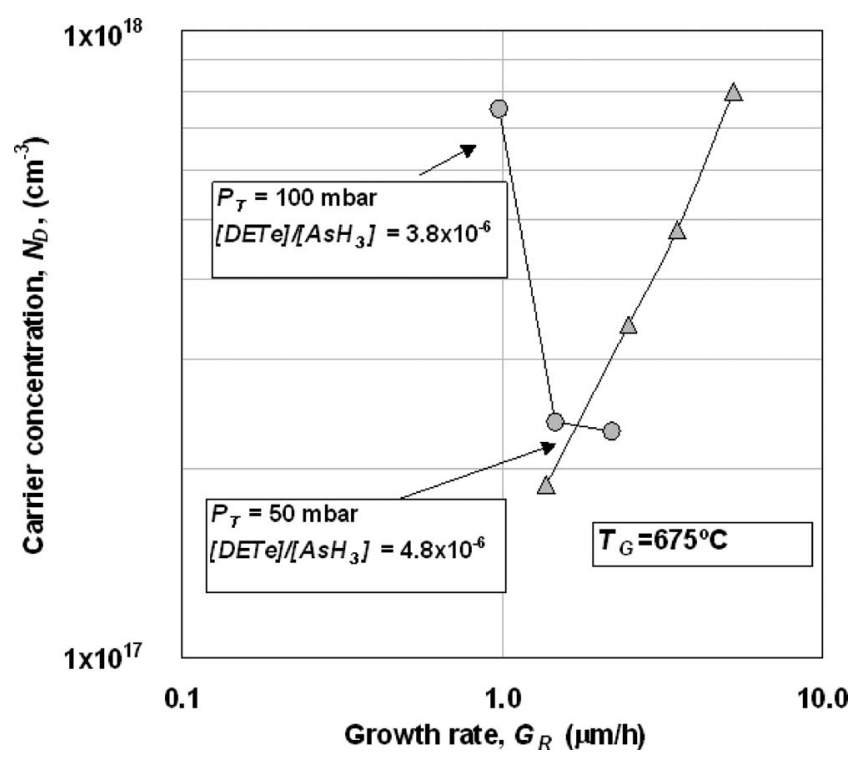

FIG. 1. Carrier concentration $\left(N_{D}\right)$ vs growth rate $\left(G_{R}\right)$ for different layers grown on $2^{\circ} \rightarrow(111) A$ substrates at 50 and 100 mbar.

\section{RESULTS AND DICUSSION}

\section{A. Influence of the total pressure and the growth rate on the carrier concentration}

In Fig. 1, carrier concentration $\left(N_{D}\right)$ versus growth rate $\left(G_{R}\right)$ is plotted for substrate miscut $2^{\circ} \rightarrow(111) A$ for runs carried out at 50 and 100 mbar. $\left[\mathrm{AsH}_{3}\right]$ and [DETe] have been kept constant for the two sets of experiments (50 and 100 mbar) in order to avoid effects related to the competition of Te and As for the group-V site. As can be seen, for 50 mbar, Te behaves like a typical volatile dopant $\left(N_{D} \alpha G_{R}\right)$, while for 100 mbar it behaves like a typical nonvolatile dopant $\left(N_{D} \alpha 1 / G_{R}\right)$. On the other hand, Fig. 2 compares the influence of the substrate misorientation by plotting carrier concentration $\left(N_{D}\right)$ versus growth rate $\left(G_{R}\right)$ for both types of

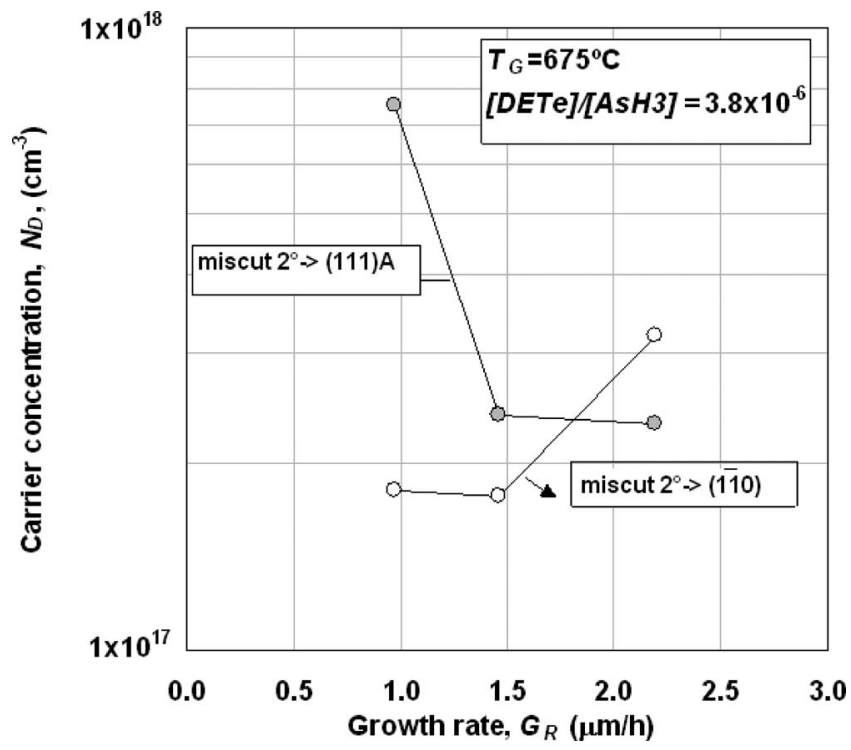

FIG. 2. Carrier concentration $\left(N_{D}\right)$ vs growth rate $\left(G_{R}\right)$ for different layers grown at $P_{T}=100 \mathrm{mbar}$ on $2^{\circ} \rightarrow(111) A$ substrates and on $2^{\circ} \rightarrow(1 \overline{1} 0)$ substrates. substrate, in this case for growths carried out at 100 mbar and $675^{\circ} \mathrm{C}$. Therefore, the only difference between both samples in this experiment is the surface configuration resulting from the two different miscuts used. It can be seen that for substrate miscut $2^{\circ} \rightarrow(1 \overline{1} 0)$ Te seems to behave like a volatile dopant, while for $2^{\circ} \rightarrow(111) A$ substrates Te tends to behave like a nonvolatile dopant.

These experimental results (Figs. 1 and 2) cannot be explained by gas phase reactions since the As/Te ratio is kept constant for every growth carried out on the same substrate. Consequently, reactions on the surface seem to be responsible for this behavior. The change of a factor of about 3 in the net donor concentration when the growth rate is changed by about $50 \%$ (super linear effect) could be related to the decomposition of arsine that usually involves a free radical mechanism that can be very nonlinear with concentration; although this explanation cannot explain a different behavior of the Te incorporation (volatile versus nonvolatile). For this effect (shift from volatile to nonvolatile), the most likely explanation could be related to a difference in the adsorption of Te atoms on the surface depending on the substrate misorientation. Accordingly, when growing at a reactor pressure of 50 mbar the relative equilibrium vapor pressure of Te is so high that it dominates the balance between evaporation and surface adsorption. However, when the pressure is raised to 100 mbar, the relative equilibrium vapor pressure of Te drops and the volatility of $\mathrm{Te}$ diminishes, making the reactions on the surface more visible. Under these conditions the adsorption of Te atoms is favored when a substrate misorientated toward the (111)A plane is used. Consequently, more Te atoms can remain on the surface, thus changing the dopant behavior from volatile to nonvolatile. On the other hand, when substrate miscut toward the $(1 \overline{10})$ direction is used there is no significant change in the tendency of Te to stick to the surface when the $P_{T}$ is modified, and thus its volatile nature remains. The reason for the variation in the adsorption of the amount of Te atoms from one substrate to another is not presently understood. It could be related to the surface reconstruction provoked by $\mathrm{Te}$, the difference in the adsorption rate depending on the substrate miscut or a mixture of difference effects.

In summary, for a given temperature, both the reactor pressure (which influences Te relative equilibrium vapor pressure) and the surface miscut (which determines the type of reconstruction and potential adsorption sites for $\mathrm{Te}$ ) are the facts that eventually determine the volatile or nonvolatile nature of the Te dopant and thus the mechanisms that control Te incorporation into the crystal lattice, and consequently the influence of the growth rate.

\section{B. Effect of the growth temperature on the carrier concentration}

In order to verify the aforementioned results, the influence of growth temperature for different substrates, for different total pressures, and for different Te molar fractions ([DETe]) has been evaluated. The experimental data have been divided into two groups: the experimental data for 50 mbar have been plotted in Fig. 3, while the experimental data 


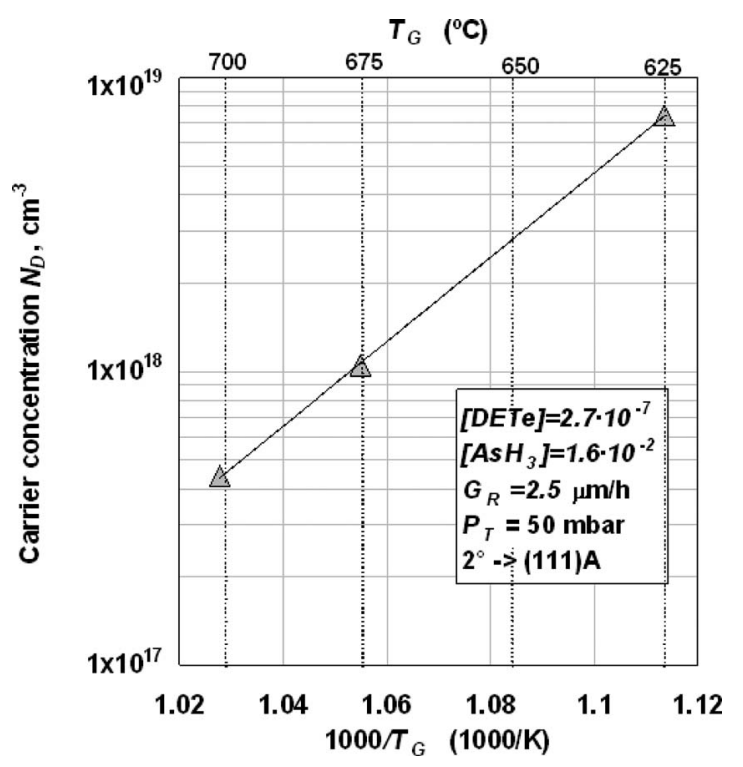

FIG. 3. Arrhenius plot for different Te-doped GaAs layers grown at $P_{T}$ $=50 \mathrm{mbar}$ on $2^{\circ} \rightarrow(111) \mathrm{A}$ substrates.

for 100 mbar are detailed in Fig. 4. For 50 mbar the growths have been carried out on substrate miscut $2^{\circ} \rightarrow(111) A$ only, using a single amount of Te $\left([\mathrm{DETe}]=2.7 \times 10^{-7}\right)$ and a growth rate of $2.5 \mu \mathrm{m} / \mathrm{h}$. On the other hand, for $P_{T}$ $=100$ mbar (Fig. 4), both substrate miscuts were used $\left[2^{\circ}\right.$ $\rightarrow(1 \overline{1} 0)$ and $\left.2^{\circ} \rightarrow(111) A\right]$ and three different [DETe] values were evaluated for a growth rate of $1.0 \mu \mathrm{m} / \mathrm{h}$. In order to assess the influence of the growth temperature in Te incorporation, the experimental data have been fitted using the Arrhenius expression included in Eq. (1), where $k_{B}$ is the Bolztmann constant, $E_{A_{-} T e}$ is the activation energy of Te, and $A$ is the pre-exponential factor,

$$
N_{D}=A \exp \left[\frac{-E_{A \_\mathrm{Te}}}{k_{B} T_{G}}\right] .
$$

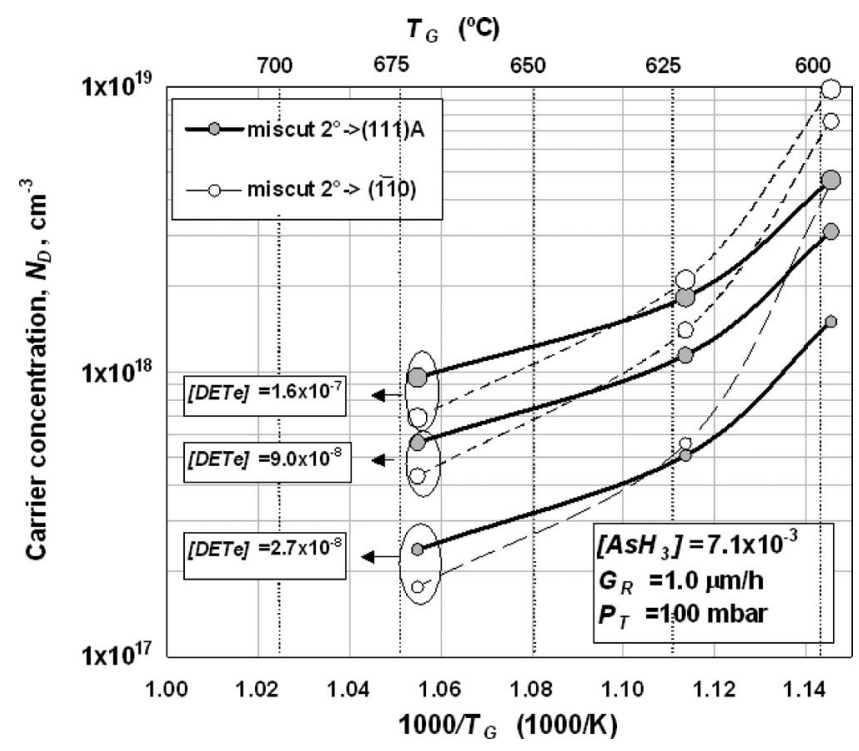

FIG. 4. Arrhenius plot for three different DETe molar fractions ([DETe] $=2.7 \times 10^{-8}, 9.0 \times 10^{-8}$, and $\left.1.6 \times 10^{-7}\right)$ and both substrates: $2^{\circ} \rightarrow(1 \overline{1} 0)$ (white dots) and $2^{\circ} \rightarrow(111) A$ (gray dots). $P_{T}=100 \mathrm{mbar}$.
TABLE I. Tellurium activation energy $\left(E_{A_{-} \mathrm{Te}}\right)$ for different substrate misorientations, growth pressures $\left(P_{T}\right)$, and DETe molar fractions ([DETe]) calculated from the experimental data in Figs. 3 and 4.

\begin{tabular}{lccc}
\hline \hline $\begin{array}{l}P_{T} \\
(\mathrm{mbar})\end{array}$ & {$[\mathrm{DETe}]$} & Substrate miscut & $\begin{array}{c}E_{A_{-} \mathrm{Te}} \\
(\mathrm{eV})\end{array}$ \\
\hline 50 & $2.7 \times 10^{-7}$ & $2^{\circ} \rightarrow(111) A$ & 6.60 \\
100 & $2.7 \times 10^{-8}$ & $2^{\circ} \rightarrow(111) A$ & 2.37 \\
100 & $9.0 \times 10^{-8}$ & $2^{\circ} \rightarrow(11) A$ & 2.37 \\
100 & $1.6 \times 10^{-7}$ & $2^{\circ} \rightarrow(111) A$ & 2.21 \\
100 & $2.7 \times 10^{-8}$ & $2^{\circ} \rightarrow(1 \overline{1} 0)$ & 4.02 \\
100 & $9.0 \times 10^{-8}$ & $2^{\circ} \rightarrow(1 \overline{1} 0)$ & 3.94 \\
100 & $1.6 \times 10^{-7}$ & $2^{\circ} \rightarrow(1 \overline{1} 0)$ & 3.80 \\
\hline \hline
\end{tabular}

First, for $P_{T}=50 \mathrm{mbar}$ (Fig. 3) the behavior of $N_{D}$ as a function of $T_{G}$ follows an Arrhenius equation with an activation energy of $E_{A_{-} \mathrm{Te}}=6.6 \mathrm{eV}$. A high value for the activation energy means that the dependence of $N_{D}$ with $T_{G}$ is strong and that the dopant is clearly volatile. These results complement the data in Fig. 1 showing that, for sufficiently low pressures, Te behaves like a classic volatile dopant for a wide range of temperatures and growth rates, for the two substrates studied. It can be seen in Fig. 4 that the data at $P_{T}$ $=100$ mbar cannot be fitted using Eq. (1). This could be related to a hidden effect, namely, the incomplete pyrolysis of the arsine at low temperatures and consequently, the decrease in As atoms. This would enhance the incorporation of $\mathrm{Te}$, since $\mathrm{Te}$ and As compete for incorporation into the group-V sublattice. Although this effect should be independent of the substrate used, the data for $600{ }^{\circ} \mathrm{C}$ have been ignored and only the highest temperature data have been used for the calculation of $E_{A_{-} \text {Te }}$, whose values are presented in Table I for the four sets of experimental data (Figs. 3 and 4). The following conclusions can be extracted from this table.

(1) The value of $E_{A_{-} \mathrm{Te}}$ for substrate miscut $2^{\circ} \rightarrow(1 \overline{1} 0)$ is higher than that for substrate miscut $2^{\circ} \rightarrow(111) A$ for the same growth conditions $\left(T_{G}, G_{R}, P_{T}\right.$, and [DETe]). This implies that the volatility of Te on surface miscut toward the $(1 \overline{1} 0)$ plane is higher than for surface miscut toward the (111)A plane. This result is coherent with the experimental data presented in Sec. III $\left(G_{R}\right.$ versus $\left.N_{D}\right)$.

(2) For both substrates $\left[2^{\circ} \rightarrow(1 \overline{1} 0)\right.$ and $\left.2^{\circ} \rightarrow(111) A\right]$ at $100 \mathrm{mbar}$, the value of $E_{A_{-} \mathrm{Te}}$ increases (i.e., is less negative) as [DETe] increases. Thus, as the growth surface becomes saturated with Te atoms, the volatility of the Te decreases and, consequently, its dependency with temperature does the same. Additionally, this effect is more visible on substrates $2^{\circ} \rightarrow(1 \overline{1} 0)$ than on substrates $2^{\circ}$ $\rightarrow(111) A$, since for the first one the volatility of Te has much more influence on the incorporation of the dopant.

(3) Comparing $P_{T}=50 \mathrm{mbar}$ and $P_{T}=100 \mathrm{mbar}$ for the same substrate, and the same amount of Te, the value of $E_{A_{-} \mathrm{Te}}$ is lower (i.e., more negative) for $P_{T}=50 \mathrm{mbar}$ than for $P_{T}=100 \mathrm{mbar}$ since as the pressure increases the volatility of the dopant decreases. 


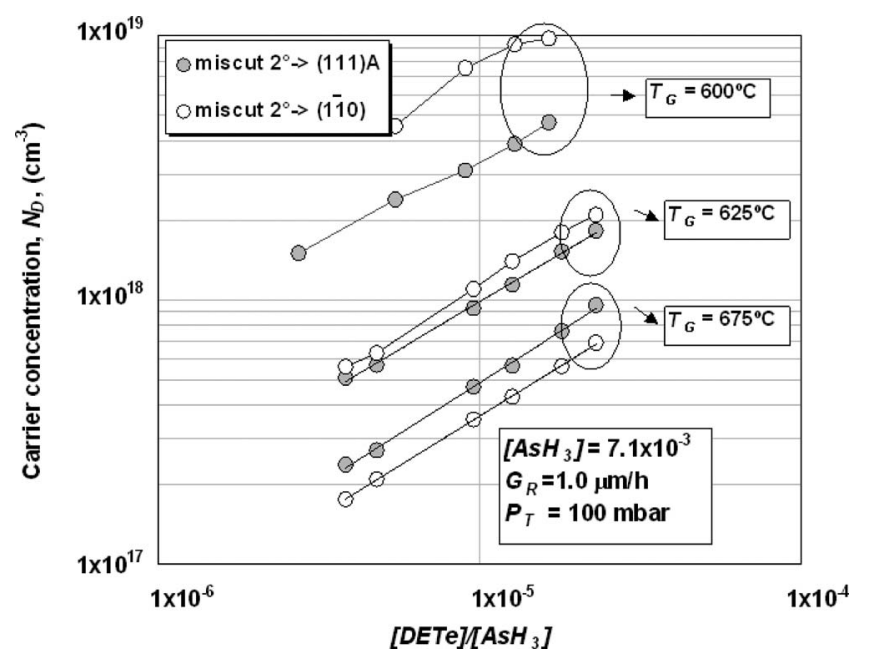

FIG. 5. Carrier concentration $\left(N_{D}\right)$ vs DETe to arsine ratio $\left([\mathrm{DETe}] /\left[\mathrm{AsH}_{3}\right]\right)$ for three different growth temperatures $\left(T_{G}=600,625\right.$, and $\left.675^{\circ} \mathrm{C}\right)$ and both substrates: $2^{\circ} \rightarrow(1 \overline{1} 0)$ and $2^{\circ} \rightarrow(111) A$.

\section{Influence of substrate misorientation on the carrier concentration}

As has been previously mentioned, the substrate orientation has a direct influence on the incorporation of Te. Depending on the substrate misorientation, the adsorption of $\mathrm{Te}$ atoms on the growth surface varies. Specifically, the adsorption of $\mathrm{Te}$ atoms is greater for substrate miscut $2^{\circ}$ $\rightarrow(111) A$ substrates than for substrate miscut $2^{\circ} \rightarrow(1 \overline{1} 0)$.

Figures 5 and 6 plot the evolution of the carrier concentration $\left(N_{D}\right)$ versus DETe to arsine ratio $\left([\mathrm{DETe}] /\left[\mathrm{AsH}_{3}\right]\right)$ for both types of substrates. Figure 5 shows these variables for different temperatures for a fixed growth rate of $1.0 \mu \mathrm{m} / \mathrm{h}$ while Fig. 6 does the same for different growth rates for a fixed growth temperature of $675{ }^{\circ} \mathrm{C}$. In principle, it is expected that carrier concentration $\left(N_{D}\right)$ becomes higher for a volatile dopant as the temperature $\left(T_{G}\right)$ decreases or the growth rate $\left(G_{R}\right)$ increases. By analyzing Fig. 5 it can be

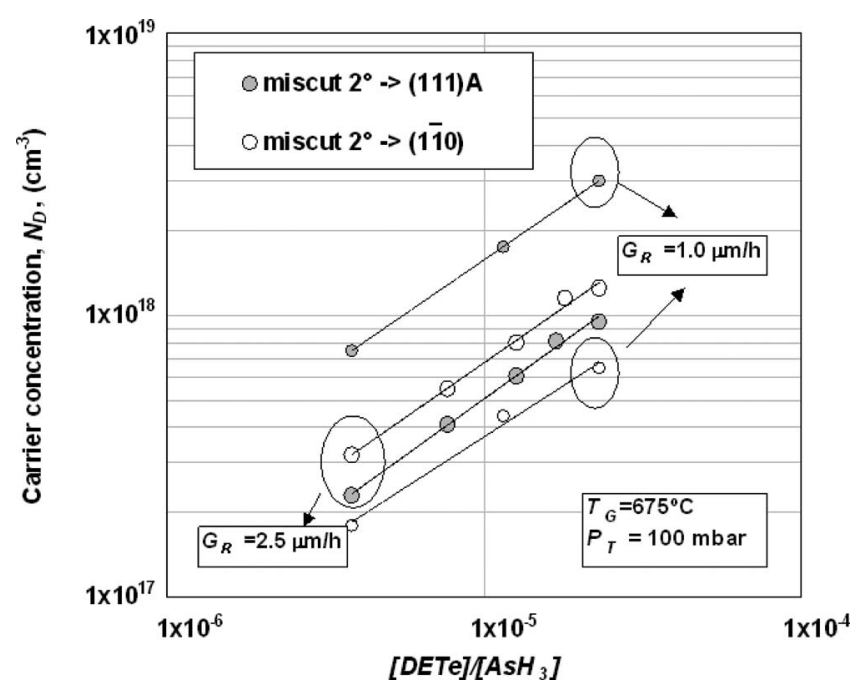

FIG. 6. Carrier concentration $\left(N_{D}\right)$ vs DETe to arsine ratio $\left([\mathrm{DETe}] /\left[\mathrm{AsH}_{3}\right]\right)$ for two different growth rates $\left(G_{R}=1.0\right.$ and $\left.2.5 \mu \mathrm{m} / \mathrm{h}\right)$ and both substrates: $2^{\circ} \rightarrow(1 \overline{1} 0)$ and $2^{\circ} \rightarrow(111) A$. seen that for the lowest temperature $\left(T_{G}=600{ }^{\circ} \mathrm{C}\right), N_{D}$ is higher for substrate miscut $2^{\circ} \rightarrow(1 \overline{1} 0)$ than for substrate miscut $2^{\circ} \rightarrow(111) A$, for the intermediate temperature $\left(T_{G}\right.$ $=625^{\circ} \mathrm{C}$ ) the carrier concentration is roughly equal for both miscuts, while for the highest temperature in the range $\left(T_{G}\right.$ $=675{ }^{\circ} \mathrm{C}$ ) the tendency is reversed, and substrate miscut $2^{\circ} \rightarrow(111) A$ produces a higher value of $N_{D}$. This result is unusual since for most dopants the variation in doping levels with the substrate misorientation does not change with the temperature. ${ }^{13,14}$ For both substrates, a decrease in temperature produces an increase in carrier concentration. However, this increase is very steep in substrate miscut $2^{\circ} \rightarrow(1 \overline{1} 0)$, where a decrease of $75{ }^{\circ} \mathrm{C}$ produces an increase in doping by a factor of 20, and far less intense in substrate miscut $2^{\circ}$ $\rightarrow(111) A$, where the same decrease in temperature increases the doping sixfold. This is again exactly the evolution expected for Te as a volatile dopant [substrate miscut toward the $(1 \overline{1} 0)$ plane] with a strong temperature dependence and for Te as a nonvolatile dopant with a much weaker temperature dependence [substrates miscut toward the (111)A plane].

Regarding the influence of the growth rate (Fig. 6), for the highest value of $G_{R}(2.5 \mu \mathrm{m} / \mathrm{h}), N_{D}$ is higher for substrate miscut $2^{\circ} \rightarrow(1 \overline{1} 0)$ than for substrate miscut $2^{\circ}$ $\rightarrow(111) A$, while for the lowest growth rate $\left(G_{R}=1 \mu \mathrm{m} / \mathrm{h}\right)$ the tendency is reversed, and substrate miscut $2^{\circ} \rightarrow(111) A$ produces a higher value of $N_{D}$. Again, this result is unusual since for most dopants the variation in doping levels with the substrate misorientation does not change with the growth rate $^{12,13}$ (i.e., when a given orientation produces a higher incorporation of dopant, this trend remains no matter the value of the growth rate). To explain the evolution in Fig. 6, we have to consider again a change between volatile to nonvolatile dopant depending on the substrate used.

For instance, for substrate miscut $2^{\circ} \rightarrow(111) A$, which have been determined (Fig. 2) to favor the nonvolatile behavior of Te for this set of growth conditions $\left(T_{G}=675{ }^{\circ} \mathrm{C}\right.$, $\left.P_{T}=100 \mathrm{mbar}\right)$, the expected decrease in carrier concentration with growth rate can be seen. The doping attained for $G_{R}=1.0 \mu \mathrm{m} / \mathrm{h}$ is around three times higher than that reached when growing at $2.5 \mu \mathrm{m} / \mathrm{h}$. On the other hand, the same growth conditions applied on substrate miscut $2^{\circ}$ $\rightarrow(1 \overline{1} 0)$ reveal the typical behavior of a volatile dopant to which an increase in growth rate produces an increase in dopant concentration.

Additionally, it should be noted that for low growth rates $(1 \mu \mathrm{m} / \mathrm{h}), N_{D}$ is higher on the $2^{\circ} \rightarrow(111) A$ substrates than on $2^{\circ} \rightarrow(1 \overline{1} 0)$ substrates, since (a) there is more Te on the surface and (b) this low growth rate gives sufficient time for all of the diffusion processes and exchange reactions to take place. Conversely, as the growth rate is increased to $2.5 \mu \mathrm{m} / \mathrm{h}$ the situation is reversed. In this case, the $N_{D}$ is slightly higher for substrate miscut $2^{\circ} \rightarrow(1 \overline{1} 0)$ than for substrate miscut $2^{\circ} \rightarrow(111) A$ since high growth rates (a) favor the capture of volatile dopants and (b) hinder some of the kinetic processes needed for the incorporation of Te when it 
is wetting the surface (i.e., if we grow too fast a significant amount of Te can float on the surface without being effectively incorporated into the lattice).

\section{SUMMARY AND CONCLUSIONS}

The growth of Te-doped GaAs samples has been addressed by considering the influence of the most relevant parameters for MOVPE growth, namely, reactor pressure, growth temperature, growth rate, and substrate miscut. It has been shown that tellurium behaves like a nonvolatile dopant or a volatile dopant depending on the total pressure and the substrate misorientation for usual MOVPE growth conditions within the mass transport limited growth regime, which determines the relationship with the growth rate. This unusual behavior can be explained by means of effects carried out on the growth surface. According to the experimental data presented in this paper, when (100) GaAs miscut $2^{\circ}$ $\rightarrow(111) A$ substrates are used, the reactor pressure during growth can modulate the behavior of Te. More concretely, for very low pressures (50 mbar) Te behaves like a volatile dopant (for instance, doping concentration increases with growth rate), but when the pressure is raised to 100 mbar, the doping behavior is reversed and Te acts like a typical nonvolatile dopant (i.e., doping concentration decreases with growth rate). On the other hand, when (100) GaAs miscut $2^{\circ} \rightarrow(1 \overline{10})$ substrates are used, Te always behaves like a volatile dopant.

\section{ACKNOWLEDGMENTS}

This study has been supported by the European Commission under Contract No. SES6-CT-2003-502620 (FULL-
SPECTRUM project). The Spanish Ministerio de Educación y Ciencia has also contributed with the CONSOLIDERINGENIO 2010 program by means of the GENESIS FV project (Grant No. CSD2006-004) and also with the research projects with Reference Nos. TEC2005-02745 and TEC2004-22300-E as well as the Comunidad de Madrid under NUMANCIA program (Grant No. S-05050/ENE/0310). The Universidad Politécnica de Madrid has also contributed with the research projects with Reference Nos. A0415 and M0700204145.

${ }^{1}$ Y.-M. Houng and T. S. Low, J. Cryst. Growth 77, 272 (1986).

${ }^{2}$ S. Z. Sun, E. A. Armour, K. Zheng, and C. F. Schaus, J. Cryst. Growth 113, 103 (1991).

${ }^{3}$ M. Kamp, G. Morsch, J. Graber, and H. Luth, J. Appl. Phys. 76, 1974 (1994).

${ }^{4}$ W. M. Li, C. Y. Chen, and R. M. Cohen, J. Cryst. Growth 156, 343 (1995).

${ }^{5}$ G. B. Stringfellow, Organometallic Vapor Phase Epitaxy: Theory and Practice, 2nd ed. (Academic, New York, 1999).

${ }^{6}$ M. K. Hudait and S. B. Krupanidhi, Solid State Commun. 108, 457 (1998).

${ }^{7}$ H. Sugiura, C. Amano, and A. Yammoto, Jpn. J. Appl. Phys., Part 1 27, 269 (1988).

${ }^{8}$ B. J. Sealy, N. J. Barrett, and R. Bensalem, J. Phys. D 19, 2147 (1986).

${ }^{9}$ I. García, I. Rey-Stolle, B. Galiana, and C. Algora, J. Cryst. Growth 298, 794 (2007).

${ }^{10}$ SW. Jun, G. B. Stringfellow, A. D. Howard, C. M. Fetzer, and J. K. Shurtleff, J. Appl. Phys. 90, 6048 (2001).

${ }^{11}$ C. D. Consorte, C. Y. Fong, M. D. Watson, L. H. Yang, and S. Ciraci, Mater. Sci. Eng., B 96, 141 (2002).

${ }^{12}$ R. R. Wixom, L. W. Rieth, and G. B. Stringfellow, J. Cryst. Growth 269, 276 (2004)

${ }^{13}$ P. R. Hageman, J. Nijenhuis, M. J. Anders, and L. J. Giling, J. Cryst. Growth 170, 270 (1997).

${ }^{14}$ M. Kondo and T. Tanahashi, J. Cryst. Growth 145, 390 (1994). 